\title{
$\mathbf{N}_{2} \mathbf{O}_{4}$ 环境下液体火箭发动机涡轮泉 机械密封浸渍石墨的磨损机理研究*
}

\author{
王建磊 $^{1}$ 张 琛 $^{1}$ 王晓虎 $^{1}$ 王栋平 $^{1}$ 李建克 $^{2}$ \\ 贾 谦 $^{3}$ 陈润霖 $^{1}$ 崔亚辉 $^{1}$
}

(1. 西安理工大学机械与精密仪器工程学院 西安 710048;

2. 中国航天科技集团公司西安航天动力研究所 西安 710100 ;

3. 西安交通大学现代设计及转子轴承系统教育部重点实验室 西安 710049)

摘要: 针对液体火箭发动机浴轮百机械密封浸渍石墨在 $\mathrm{N}_{2} \mathrm{O}_{4}$ 环境下磨损量较大的现象, 以宏观试验与微观检测相结合的方 法探索其产生的机理。对机械密封石墨的磨损表面进行了电镜观测，根据磨损形貌对正常磨损区与异常磨损区进行了区分， 并对异常磨损的诱因进行了假设。提出了石墨表面的树脂腐蚀模型与孔隙气蚀模型, 设置了包含静态腐蚀及动态磨损的试验 流程以验证树脂腐蚀和孔隙气蚀的作用结果, 分别在水及 $\mathrm{N}_{2} \mathrm{O}_{4}$ 环境下进行试验, 并通过扫描电镜及红外光谱分析等手段观 测了试验前后石墨表面的微观组织变化, 介质试验后气孔平均直径为 $33 \mu \mathrm{m}$, 为试验前的 2 倍。研究结果表明: $\mathrm{N}_{2} \mathrm{O}_{4}$ 环境并 不会造成石墨浸渍物酚醛树脂的腐蚀, 造成石墨磨损量较大的诱因是气相 $\mathrm{N}_{2} \mathrm{O}_{4}$ 在石墨表面孔隙内部破裂导致的气蚀, 气蚀 后石墨的磨损可达数十微米量级。针对减少浸渍石墨的气蚀, 给出了 3 项制备工艺的改进措施。本文的研究成果也可为其他 高速旋转机械的机械密封石墨磨损研究及选材提供参考。

关键词: 火箭发动机; 浴轮原; 机械密封; 磨损; 气蚀

中图分类号: TH117

\section{Wear Mechanism of Liquid Rocket Engine Turbopump Mechanical Seal Graphite Surface in the $\mathrm{N}_{2} \mathrm{O}_{4}$ Environment}

\author{
WANG Jianlei $^{1} \quad$ ZHANG Chen $^{1} \quad$ WANG Xiaohu ${ }^{1}$ WANG Dongping $^{1} \quad$ LI Jianke $^{2}$ \\ JIA Qian ${ }^{3}$ CHEN Runlin ${ }^{1} \quad$ CUI Yahui ${ }^{1}$ \\ (1. School of Machinery and Precision Instrument Engineering, Xi' an University of Technology, Xi'an 710048; \\ 2. Aerospace Propulsion Institute, China Aerospace Science and Technology Corporation, Xi'an 710100; \\ 3. Key Laboratory of Modern Design and Rotor Bearing System, Ministry of Education, \\ Xi'an Jiaotong University, Xi'an 710049)

\begin{abstract}
In view of the phenomenon that the mechanical seal impregnated graphite has a large amount of wear in $\mathrm{N}_{2} \mathrm{O}_{4}$ environment, the mechanism by combining the macroscopic test and microscopic examination is explored. The wear surface of graphite in mechanical seal is observed by electron microscope. The normal wear zone and abnormal wear zone are distinguished according to wear morphology, and the cause of abnormal wear is assumed. The resin corrosion model and pore cavitation model of graphite surface are proposed. The test process of static corrosion and dynamic wear is set up to verify the effect of resin corrosion and pore cavitation. The experiments are carried out in water and $\mathrm{N}_{2} \mathrm{O}_{4}$ environment, and the microstructure changes of graphite surface are observed by scanning electron microscope and infrared spectrum analysis. The average pore diameter after the medium test is $33 \mu \mathrm{m}$, which is twice that before the test. The results show that the $\mathrm{N}_{2} \mathrm{O}_{4}$ environment does not cause the corrosion of graphite impregnated phenolic resin, and the cause of larger wear of graphite is cavitation caused by gas phase $\mathrm{N}_{2} \mathrm{O}_{4}$ rupture inside the graphite surface pores. The wear of graphite after cavitation can reach the order of tens of microns. In order to reduce cavitation erosion of impregnated graphite, the improvement measures of three preparation processes are given. The research results can also provide
\end{abstract}

* 西安理工大学科技创新计划资助项目(2014CX006)。20180411 收到初稿, 20181026 收到修改稿 
reference for graphite wear research and material selection of mechanical seals of other high-speed rotating machinery.

Key words: rocket engine; turbopump; mechanical seal; wear; cavitation

\section{0 前言}

火箭发动机涡轮㬌是液体火箭的动力来源, 机 械密封是火箭发动机浴轮洜的一个重要组件, 其主 要功能是阻止燃烧室内部的燃剂和助燃剂混合, 避 免危险事故的发生, 因此机械密封直接决定浴轮原 的安全运行 ${ }^{[1-2]}$ 。机械密封工作中常存在密封腔温度 过高、压力引起的密封零件变形及静环磨损等问题, 其中静环磨损是最常见的一种 ${ }^{[3]}$ 。

针对机械密封的磨损现象, 很多研究者做了大 量工作。在磨损模型的研究方面, 丁雪星等 ${ }^{[4]}$ 结合 热传导理论及概率统计建立了一种针对机械密封摩 擦界面温度分布的分形模型, 该模型支持对温度分 布规律及影响因素分析。李克斯等 ${ }^{[5]}$ 提出了一种基 于先验知识的状态评估模型, 该模型可用于对密封 端面的磨损状态进行评估。在磨损仿真分析的研究 方面, 张晓东等 ${ }^{[6]}$ 针对机械密封动、静环相互滑动 的过程, 通过理论分析及有限元法对机械密封动静 环之间的摩擦磨损进行了模拟。王悦迏等 ${ }^{[7]}$ 针对机 械密封建立了动、静环摩擦副有限元仿真模型, 分 析了端面变形与泄漏的关系, 仿真分析得出的泄漏 量与设计值接近, 并指出了过盈配合在机械密封性 能分析中有重要的影响。赵伟刚等 ${ }^{[8]}$ 对比了机械密 封静环磨损前后的差异，并通过仿真分析了密封端 面受力及温升对其磨损的影响, 指出摩擦产生的大 量热会影响静环材料的性能, 进而加剧密封端面静 环的磨损。李双喜等 ${ }^{[9]}$ 研究了机械密封的端面接触 应力对其磨损的影响, 建立了动环、静环等的完整 模型, 仿真分析的结果表明工作压力的增加会导致 密封端面的局部接触, 从而因过大压力导致密封的 过度磨损。在密封摩擦副材料的性能改进研究方面, 王建否等 ${ }^{[10]}$ 针对低温环境下的机械密封摩擦副, 制 备了一种具有低摩擦系数的纳米尺度耐磨涂层, 并 对该涂层进行了液氮环境下的摩擦磨损性能测试, 为低温工况下的机械密封选材提供了重要参考。贾 谦等 ${ }^{[11]}$ 针对静环石墨材料的磨损问题, 制备了三种 石墨化度不同的浸渍石墨, 对其在腐蚀环境下的摩 擦学性能进行了测试, 为腐蚀环境下的静环材料的 性能改进提供了试验依据。

浸渍石墨是机械密封常用的静环材料, 浸渍石 墨具有良好的自润滑性及耐磨性, 适用于高速重载 场合。在对火箭发动机浴轮洜机械密封进行试验时
常发现，在其他工况相同时，浸渍石墨在 $\mathrm{N}_{2} \mathrm{O}_{4}$ 环境 下的磨损远大于在水润滑介质的环境下 ${ }^{[12]}$ 。因此有 必要探究在 $\mathrm{N}_{2} \mathrm{O}_{4}$ 环境下浸渍石墨磨损量增大的原 因。机械密封磨损机理研究涉及机械工程、材料科 学及应用物理等多学科知识, 密封副材料性能改进 需从宏观和微观两个方面结合起来进行。本文对机 械密封石墨磨损件进行微观分析，建立石墨磨损模 型, 通过试验进行模型验证, 以得出磨损机理。

\section{1 机械密封的使用背景及磨损件分析}

\section{1 机械密封结构及使用工况}

本文研究对象是一种接触式波纹管机械密封, 该类机械密封常用于液体火箭发动机涡轮永轴端密 封。如图 1 为其结构简图, 该密封主要由动环、静 环和波纹管三部分组成。动环的材料为 $9 \mathrm{Cr} 18$, 其 固定在转子上随着转子一起旋转; 静环的主要材料 为酚醛树脂浸渍石墨，石墨安装在金属底座上并与 波纹管相连 ${ }^{[13]}$ 。静环外部介质为具有腐蚀性的 $\mathrm{N}_{2} \mathrm{O}_{4}, \mathrm{~N}_{2} \mathrm{O}_{4}$ 为火箭发动机燃烧室提供氧气。静环内 部介质为火箭燃料 $\mathrm{C}_{2} \mathrm{H}_{8} \mathrm{~N}_{2}$ (偏二甲肼), 其将和 $\mathrm{N}_{2} \mathrm{O}_{4}$ 在燃烧室结合燃烧, 产生使火箭升空的推力。该类 机械密封的主要作用就是阻止以上两种介质在浴轮 洜中发生混合，确保浴轮洜安全运转。

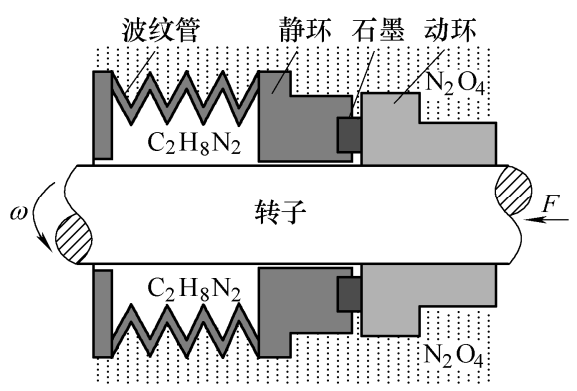

图 1 涡轮䈋机械密封的结构简图

表 1 中列出了本文机械密封的工况及结构参 数, 其工作转速较高, 工作压力略高于大气压, 工

表 1 机械密封工况及结构参数

\begin{tabular}{cc||cc}
\hline 工况参数 & 数值 & 结构参数 & 数值 \\
\hline 涡轮㬌转速 $n /$ & $\geqslant 30000$ & 动环外径 $D / \mathrm{mm}$ & 40 \\
$(\mathrm{r} / \mathrm{min})$ & & 静环外径 $D / \mathrm{mm}$ & 50 \\
工作温度 $T /{ }^{\circ} \mathrm{C}$ & $20 \sim 25$ & 静环内径 $d / \mathrm{mm}$ & 25 \\
工作压力 $p / \mathrm{MPa}$ & $0.8 \sim 0.9$ & 动环材料 & $9 \mathrm{Cr} 18$ \\
工作时间 $t / \mathrm{s}$ & $\leqslant 2400$ & 静环材料 & 浸渍石墨 \\
线速度 $v /(\mathrm{m} / \mathrm{s})$ & $50 \sim 80$ & & \\
\hline
\end{tabular}


作温度接近常温。与一般的机械密封相比, 火箭发 动机浴轮㬌机械密封对摩擦磨损、抗冲击载荷及高 速稳定性等指标要求更高 ${ }^{[14]}$ 。

\section{2 石墨磨损表面的微观组织检测与分析}

机械密封的研制一般根据手册和经验进行, 本 文机械密封的研制过程分 8 个环节, 流程如图 2 所 示, 其中摩擦学性能检测和全尺寸台架试验这两个 环节尤为重要。台架试验的目的是模拟真实工况来 检测机械密封是否符合设计要求, 为其与发动机联 试做最后的准备。本文机械密封在 $\mathrm{N}_{2} \mathrm{O}_{4}$ 介质试验时 静环的石墨表面常伴有较大面积磨损, 为增加密 封可靠性, 需要解释产生这种现象的机理, 同时 也便于通过理论对石墨材料或密封的设计参数进 行改进。

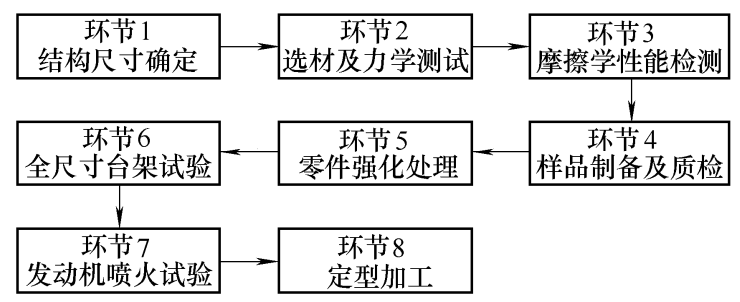

图 2 机械密封研制的流程

图 3a 为某次 $\mathrm{N}_{2} \mathrm{O}_{4}$ 介质台架试验后的机械密封 静环, 试验中静环的石墨表面磨损严重, 引起了密 封的失效。而在前期进行的水介质试验时, 此类现 象并未发生。对静环的石墨表面进行电镜观测时发 现其具有三类损伤特征, 分别是均匀磨损(区域 1)、 微孔群(区域 2)及巨型孔隙(区域 3)。

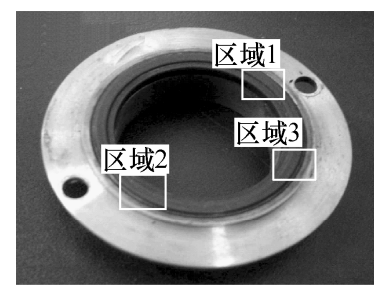

(a) 石墨静环

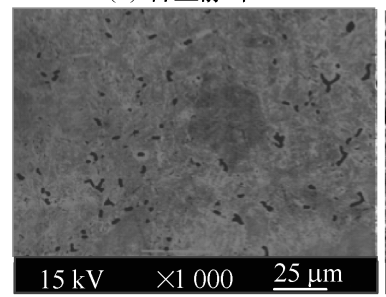

(c) 区域 2

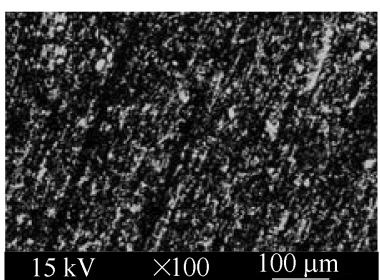

(b) 区域 1

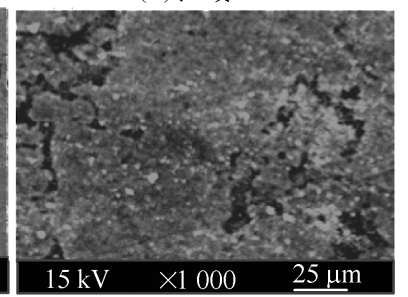

(d) 区域 3
图 3 机械密封的石墨表面

图 $3 b$ 为区域 1 处的电镜图片, 此处石墨表面出 现深度较浅且细长的梨沟状磨痕, 且分布均匀, 其 形貌符合接触面微凸体造成的正常磨损。图 3c 为区 域 2 处的电镜图片, 此处出现了直径约 $2 \mu \mathrm{m}$ 的微孔
群, 这些微孔形状规律且分布均匀, 形貌上符合气 蚀现象。因为 $\mathrm{N}_{2} \mathrm{O}_{4}$ 的沸点较低, 常温下常有气相存 在, 此处微孔应为气相 $\mathrm{N}_{2} \mathrm{O}_{4}$ 气蚀导致。图 $3 \mathrm{~d}$ 为区 域 3 处的电镜图片, 此处出现了尺寸在 $20 \mu \mathrm{m}$ 以上 且形状不规则的大块凹坑, 凹坑较深, 应是石墨表 面因外力导致的塌陷或剥落, 属于异常磨损现象。 对此处异常磨损区域进行了能谱扫描, 未发现金属 成分，排除了此处磨损是因为金属硬物侵入造成。

根据以上分析, 区域 3 处的异常磨损应为导致 试验中石墨静环密封失效的主要原因。而表面形貌 只是异常磨损的外在表现, 要探究其产生原因就需 要寻找导致此处石墨容易产生脱落的内因。

\section{3 磨损诱因的假设模型}

摩擦副设计时, 一般根据理论或经验, 给出两 表面的粗粮度, 加工时根据图纸实现粗粘度设计要 求。图 4 为机械密封摩擦副表面微观模型, 图 4a 所示为设计机械密封时动环和静环石墨的理想表 面。图 4b 所示为实际加工后的机械密封摩擦副表面 模型, 因为石墨是一种多孔材料, 加工表面会有开 放型孔隙存在, 称为开口气孔。这些气孔虽可通过 浸渍工艺填补，但仍会有气孔率在 $0.1 \% \sim 0.5 \%$ 左右 的气孔留于表面 ${ }^{[15]}$ 。本文石墨气孔中填有酚醛树 脂, 在密封工作时加入填充的树脂或者孔隙遭到破 坏就会出现较大的孔隙, 如图 4c 所示, 此时石墨内 部容易出现应力集中导致层片状或者块状的的磨损 脱落, 从而产生表面破损影响密封性能 ${ }^{[16]}$ 。因此, 根据本文异常磨损的形貌特征，初步怀疑是石墨表 面由于某种原因使得石墨本身的某些局部的表面结 构产生了变化, 使得石墨表面强度得到了弱化, 变 得疏松, 从而使其在台架试验遭遇碰磨时易产生脱 落。这种现象在进行水润轴承石墨材料的研发时也 极为常见, 例如在对核主洜用水润滑石墨轴承制备 及性能检测的数据化研究中 ${ }^{[17]}$ 。

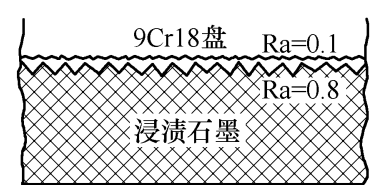

(a) 理想表面

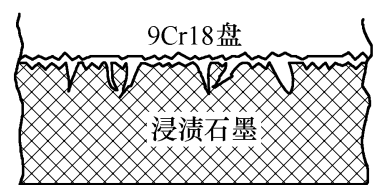

(b) 实际表面

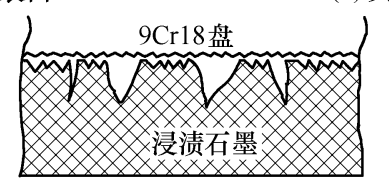

(c) 损伤表面
图 4 摩擦副表面模型

为进一步探究产生表面破损的诱因，可作两类 假设, 分别是树脂腐蚀(诱因 1)和孔隙气蚀(诱因 2)。 
由于石墨材料本身具有耐腐蚀性, 因此假设, 导致 石墨磨损量较大的诱因之一为石墨表面浸渍物酚醛 树脂被 $\mathrm{N}_{2} \mathrm{O}_{4}$ 腐蚀。图 5 为树脂腐蚀模型图, 在图 $5 \mathrm{a}$ 中 $\mathrm{N}_{2} \mathrm{O}_{4}$ 液体位于石墨与密封动环 $(9 \mathrm{Cr} 18)$ 界面之 间, 树脂浸渍填充了石墨表面的孔隙, 使得石墨表 面变得致密。假如 $\mathrm{N}_{2} \mathrm{O}_{4}$ 液体腐蚀了树脂, 就会出现 如图 5b 所示的特征, 此时石墨表面填充的树脂被大 量腐蚀脱落, 从而使得石墨表面出现较大且深入的 孔隙, 此时石墨的强度弱化, 容易诱发磨损。

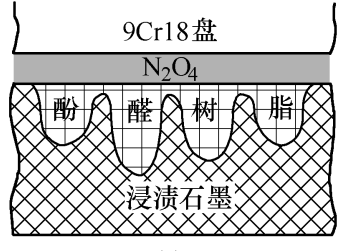

(a)

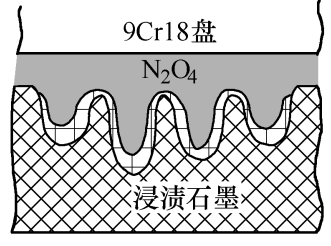

(b)
图 5 树脂腐蚀模型

另外, 由于 $\mathrm{N}_{2} \mathrm{O}_{4}$ 在常温下容易气化, 因此密封 工作时会有气相 $\mathrm{N}_{2} \mathrm{O}_{4}$ 存在, 在一定工况下 $\mathrm{N}_{2} \mathrm{O}_{4}$ 气 泡会汇聚在石墨表面, 并且从小聚大, 极易渗入石 墨表面孔隙中, 如图 6a 所示。一旦 $\mathrm{N}_{2} \mathrm{O}_{4}$ 气泡在石 墨孔隙中发生爆裂, 其爆裂产生能量就对石墨内部 产生破坏, 造成深入型气蚀, 如图 $6 \mathrm{~b}$ 所示。此时石 墨表面会呈现开放型损伤, 甚至将离散气孔贯通, 形成尺寸较大的连通区域, 对石墨表面的破坏极大。

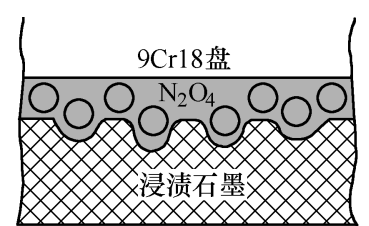

(a)

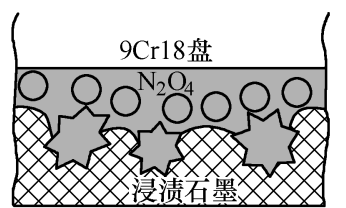

(b)
图 6 孔隙气蚀模型

\section{2 对磨损诱因的验证试验}

\section{1 试验设计}

拟通过对宏观试验结合微观检测来验证造成异 常磨损的诱因。因为树脂腐蚀(诱因 1)与孔隙气蚀 (诱因 2)有可能同时发生, 为区分两种诱因的作用效 果, 验证试验需要进行解耦设计。本文采用分步试 验来分别考察树脂腐蚀与孔隙气蚀现象, 试验流程 如图 7 所示。

试验分别在水和 $\mathrm{N}_{2} \mathrm{O}_{4}$ 环境下进行, 目的是为了 解释在 $\mathrm{N}_{2} \mathrm{O}_{4}$ 环境下石墨磨损往往远大于在水环境 下的原因。试验首先在验证诱因 1 , 对石墨进行静 态腐蚀试验, 以验证 $\mathrm{N}_{2} \mathrm{O}_{4}$ 是否会腐蚀石墨表面的树 脂, 如未腐蚀, 就不存在耦合问题, 仅需要进行下

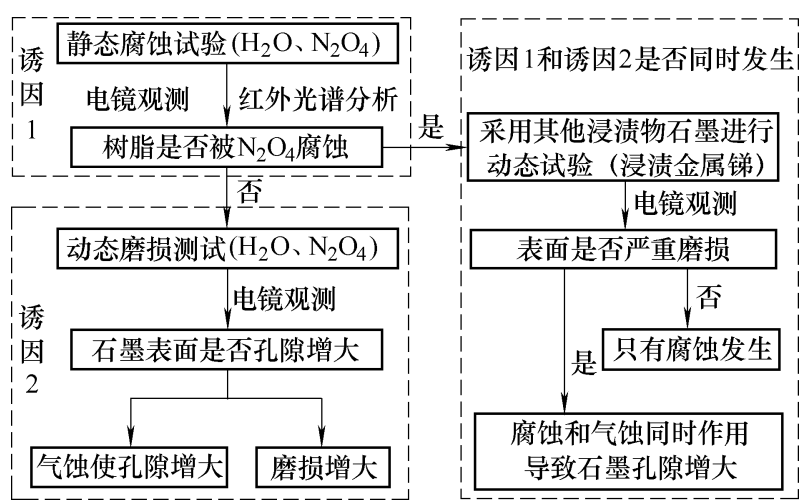

图 7 试验设计流程图

一步动态磨损试验以验证诱因 2; 如验证诱因 1 时 发现树脂被 $\mathrm{N}_{2} \mathrm{O}_{4}$ 腐蚀, 则需要更换其他浸渍物为耐 $\mathrm{N}_{2} \mathrm{O}_{4}$ 腐蚀材料的石墨(如硅化石墨或浸锑石墨)进行 动态磨损试验以验证诱因 2 是否与诱因 1 相伴发生, 此时所用石墨的气孔率指标需和上步试验所使用的 石墨材料相近。

静态腐蚀试验采用常用的浸泡方法进行, 试验 参照《GB/T10124-88 金属材料试验室均匀腐蚀全 浸试验方法》, 该类试验常被用于在腐蚀介质环境下 服役的机械产品的性能检验, 例如对石油管道静密 封材料的耐腐检测，获取相关腐蚀数据，为预测构 件剩余寿命提供数据源 ${ }^{[18]}$ 。本文试验的目的是验证 $\mathrm{N}_{2} \mathrm{O}_{4}$ 是否会腐蚀石墨中的树脂造成石墨表面疏松, 从而引发石墨磨损, 先前的静态浸泡试验发现, 浸 泡时间超过 $1 \mathrm{~h}$ 就可以达到石墨与介质的充分接触, 评价的指标是石墨的增重量。本文试验设置的时间 为 $10 \mathrm{~h}$, 已经做到了石墨与介质的充分接触。试验 是在环境温度为 $25^{\circ} \mathrm{C}$ 下进行的, 试验前先对试验件 进行超声波清洗, 试验时将试验件放入密闭容器中, 然后将介质注入容器中。由于 $\mathrm{N}_{2} \mathrm{O}_{4}$ 介质具有毒性, 因此所选择容器必须气密性良好, 且设置有气体检 测装置。本文中 $\mathrm{N}_{2} \mathrm{O}_{4}$ 的气相实现是被动的, 在试验 中提供的是液态的 $\mathrm{N}_{2} \mathrm{O}_{4}$, 气化的 $\mathrm{N}_{2} \mathrm{O}_{4}$ 是在试验过 程中产生的。由于常温下 $\mathrm{N}_{2} \mathrm{O}_{4}$ 介质容易气化, 为了 使其保持为液态, 需要安装压力调控装置, 通过压 力调控装置将试验压力设定为 $1.0 \mathrm{MPa}$ 。

动态磨损试验是在前文中提到的机械密封研制 环节 6 中的台架装置上进行的。机械产品的台架一 般试验可分为长期运转试验和短期强化试验两类, 其中短期强化试验多用于运转的设备, 例如对核主 洜水润滑轴承石墨材料的磨损寿命测试常采用较为 节省试验周期的短时强化试验, 试验时的载荷和转 速都比轴承实际工作时大 ${ }^{[19]}$ 。本文中浴轮泵机械密 封的实际工作时间较短, 因此为了验证石墨材料的 磨损性能采用延长工作时间方法，试验时间取实际 
工作时长的 10 倍(约 $40 \mathrm{~min}$ )进行。试验时首先将试 验件安装在试验台上, 然后将介质通入密封腔体中, 进行动态模拟测量。试验分为两组进行, 试验介质 分别为水和 $\mathrm{N}_{2} \mathrm{O}_{4}$, 两组试验的压力、转速、轴向力 等参数相同。

\section{2 试验材料}

本文机械密封的石墨材料为浸渍酚醛树脂石 墨, 其参数如表 2 所示。该石墨具有很强的抗压强 度、抗折强度等指标。石墨原材料中含 $80 \%$ 的焦炭 和 $10 \%$ 的炭黑, 其颗粒度较小, 保证了石墨在制备 完成后具有较好的性能, 来满足使用要求。

\section{表 2 石墨参数表}

\begin{tabular}{cc||cc}
\hline 参数 & 数值 & 参数 & 数值 \\
\hline 抗压强度 $\sigma_{b c} / \mathrm{MPa}$ & 178.48 & 弹性模量 $E / \mathrm{GPa}$ & 3.8 \\
抗折强度 $f c f / \mathrm{MPa}$ & 72.53 & 石墨化度 $g(\%)$ & $2.7 \sim 3.2$ \\
肖氏硬度 $H S / \mathrm{mm}$ & 93.2 & 原材料 & 焦炭/炭黑 \\
电导率 $G /(\mathrm{S} / \mathrm{m})$ & 6.5 & 颗粒度 $/ \mu \mathrm{m}$ & 200 \\
\hline
\end{tabular}

本文试验使用的石墨试验件采用相同石墨原材 料及加工工艺制得, 图 8a 为用于静态腐蚀试验所用 的石墨环实物图; 如图 $8 \mathrm{~b}$ 为进行动态磨损试验使用 的石墨静环实物图, 其为石墨环安装在静环座上制 得, 其结构便于组装到试验台上。

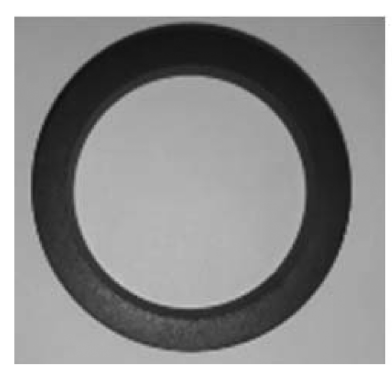

(a) 石墨环

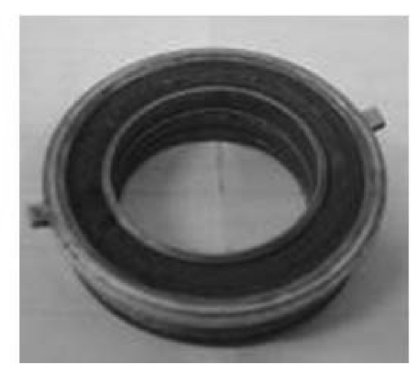

(b) 密封静环
图 8 试验件实物图

本文设计的静态腐蚀试验和动态磨损测试, 均为 对比试验。静态腐蚀试验中两组浸泡液以及动态磨损 试验中两组润滑介质, 分别为去离子水和液态 $\mathrm{N}_{2} \mathrm{O}_{4}$ 。 $\mathrm{N}_{2} \mathrm{O}_{4}$ 的沸点为 $21.2{ }^{\circ} \mathrm{C}$ 接近常温, 因此在试验中需要 控制试验环境温度以及压力来保证 $\mathrm{N}_{2} \mathrm{O}_{4}$ 为液态。

\section{3 试验设备}

静态浸泡腐蚀试验的试验设备为两个透明、容 积相同的密闭容器, 一个进行介质为水的腐蚀试验, 一个进行介质为 $\mathrm{N}_{2} \mathrm{O}_{4}$ 的腐蚀试验。容器安装有加压 装置、气体检测装置以及温度调控装置。其中加压 装置可调控压力范围为 $0.5 \sim 1.0 \mathrm{MPa}$, 目的是控制 两组试验压力环境相同和使 $\mathrm{N}_{2} \mathrm{O}_{4}$ 恒定为液态; 气体 检测装置主要是针对 $\mathrm{N}_{2} \mathrm{O}_{4}$ 容易气化产生有毒气体 而设的, 其目的是实时检测试验过程中是否有气体
泄漏; 温度调控装置可以控制温度范围为 20 $25^{\circ} \mathrm{C}$, 其精度为 $\pm 0.1^{\circ} \mathrm{C}$, 可模拟试验件在工作环境 中的温度, 且保证两组试验的试验温度相同。

动态磨损试验所使用的设备是机械密封试验 台, 该试验台可进行 1：1 尺寸的机械密封件润滑状 态下的运转试验, 试验时可模拟实际工作压力、转 速及轴向力等参数, 试验台简图如图 9 所示。该试 验台主要由驱动模块、加压模块及润滑模块等组成。 驱动模块由驱动电动机和齿轮箱组成, 加压模块通 过空气压缩机向腔体内部提供试验压力, 润滑模块 的介质存储箱为腔体内提供液态介质, 两相流检测 装置检测试验是否会产生气态介质。试验转速通过 在转轴的尾端布置电浴流传感器获得。轴向力由电 磁加载装置施加, 该电磁加载装置的设计理念曾被 成功应用于核主泵水润滑轴承可靠性强化及寿命预 测测试中 ${ }^{[20]}$, 在本文中该加载装置可提供 $50 \mathrm{~N}$ 的 轴向力。

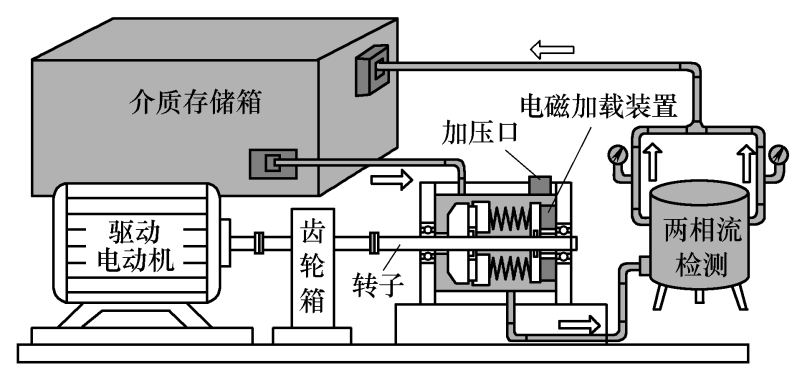

图 9 机械密封试验台简图

\section{3 试验结果及讨论}

\section{1 静态腐蚀测试}

静态浸泡后, 对试验件进行超声清洗、烘干后 进行了扫描电镜观测, 电镜图像如图 10 所示。图 $10 \mathrm{a}$ 和图 $10 \mathrm{~b}$ 分别为水环境静态腐蚀测试前后的石 墨表面, 从图中可以看出浸泡前后石墨表面孔隙的 尺寸与分布无明显变化, 说明水未对石墨的酚醛树 脂填充物产生影响。图 10c 和图 $10 \mathrm{~d}$ 分别是介质为 $\mathrm{N}_{2} \mathrm{O}_{4}$ 环境静态腐蚀前后的石墨表面, 通过观察发现 其表面均无明显变化。

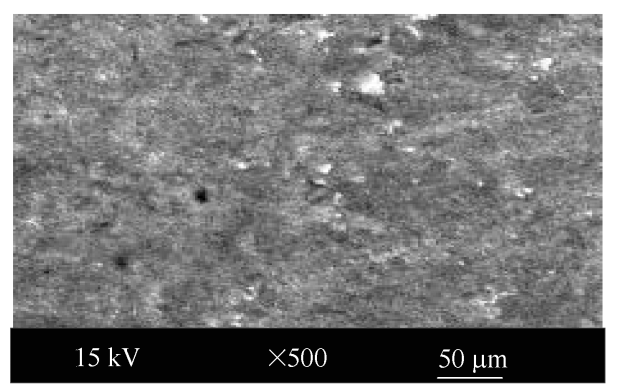

(a) 水介质浸泡前 


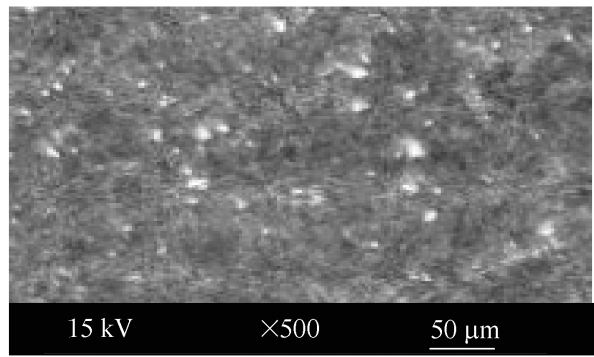

(b) 水介质浸泡后

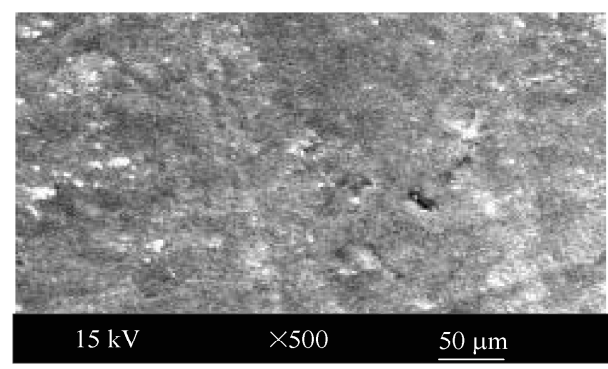

(c) $\mathrm{N}_{2} \mathrm{O}_{4}$ 介质浸泡前

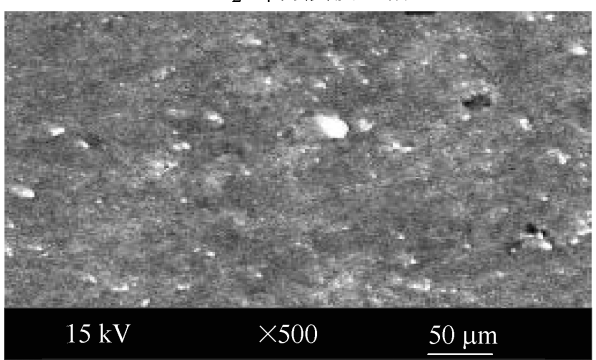

(d) $\mathrm{N}_{2} \mathrm{O}_{4}$ 介质浸泡后

图 10 静态浸泡腐蚀试验电镜图

对电镜照片中石墨表面进行二值化处理, 定量 统计了孔隙率等指标, 数值如表 4 所示。从表中可 以看出, 在水和 $\mathrm{N}_{2} \mathrm{O}_{4}$ 试验前后其表面孔隙率以及气 孔平均直径的数值都很接近。

表 4 静态腐蚀石墨表面孔隙率表

\begin{tabular}{ccc}
\hline 名称 & 表面孔隙率 $\xi(\%)$ & 气孔平均直径 $d / \mu \mathrm{m}$ \\
\hline 水介质浸泡前 & 0.36 & 13 \\
水介质浸泡后 & 0.32 & 16 \\
$\mathrm{~N}_{2} \mathrm{O}_{4}$ 介质浸泡前 & 0.41 & 11 \\
$\mathrm{~N}_{2} \mathrm{O}_{4}$ 介质浸泡后 & 0.48 & 18 \\
\hline
\end{tabular}

根据以上对静态浸泡试验石墨表面的电镜图和 孔隙统计数据可以得出: $\mathrm{N}_{2} \mathrm{O}_{4}$ 未对石墨孔隙填充物 酚醛树脂产生破坏性腐蚀。为了更进一步证实这一 判断, 又对石墨表面进行了红外光谱检测。红外光 谱可检测石墨表面树脂具有的化学键及官能团, 不 同化学键吸收频率不同，在图中所对应峰值不同。 因此, 假如树脂被腐蚀, 红外光谱测试结果就会不 同。图 11 为红外光谱测试结果, 图中曲线 1 是未经 浸泡试验的石墨光谱图, 曲线 2 和曲线 3 分别为水 腐蚀试验与 $\mathrm{N}_{2} \mathrm{O}_{4}$ 腐蚀试验后的石墨光谱图。对比
三条曲线形状可以发现, 与试验前相比, 水和 $\mathrm{N}_{2} \mathrm{O}_{4}$ 腐蚀试验后光谱曲线无明显吸收峰的增减变化, 因 此从另一侧面也证明了石墨的树脂浸渍物未遭到 腐蚀。

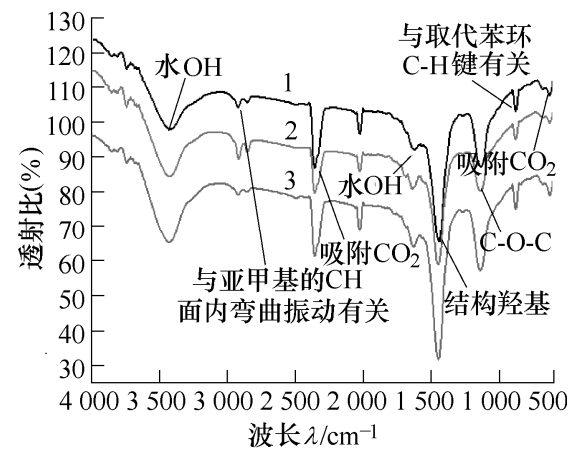

图 11 石墨表面红外光谱图

通过静态腐蚀测试可得出, 导致石墨在 $\mathrm{N}_{2} \mathrm{O}_{4}$ 环境下磨损量过大的诱因 1 在现实中不会发生。因 此, 诱因 1 和诱因 2 的耦合问题得到解耦, 下步只 需通过磨损试验去验证诱因是否会真实发生。

\section{2 动态磨损试验}

在机械密封试验台上进行了动态磨损试验后, 对石墨试验件进行了超声波清洗及烘干，试验前后 石墨表面的电镜观测图片如图 12 所示。图 12a 和图 $12 \mathrm{~b}$ 分别是水环境下试验前后的石墨表面, 图中可 以看出试验后石墨表面孔隙减小了, 原因可能是石 墨水磨后使得表面部分孔隙得到了填充。图 12c 和 图 $12 \mathrm{~d}$ 分别为 $\mathrm{N}_{2} \mathrm{O}_{4}$ 环境下试验前后的石墨表面, 图 12d 中可以看出试验后石墨表面出现了若干尺 寸较大的孔隙, 这些孔隙的长度在 $30 \mu \mathrm{m}$ 左右, 形 成的原因可能是石墨孔之间形成了连通或者石墨 表面脱落。表 5 是动态磨损测试前后石墨表面孔隙 率及气孔隙平均直径统计表, 可以看出水润滑试验 前后石墨表面孔隙并未发生明显变化; 而 $\mathrm{N}_{2} \mathrm{O}_{4}$ 润 滑试验后石墨表面孔隙率变为试验前的 10 倍以 上, 同时孔隙的平均直径也增大为试验前的 2 倍 多。在试验中两相流装置检测到有气液两相并存现 象, 一般认为 $\mathrm{N}_{2} \mathrm{O}_{4}$ 气化的主要原因是机械密封摩 擦副之间缺乏良好的润滑, 形成局部固体接触, 造 成局部过热形成的, 结合文献[8-9]中关于局部过热 和局部压力下产生磨损的相关研究结果, 本文推断 认为在 $\mathrm{N}_{2} \mathrm{O}_{4}$ 环境下, 造成液体火箭发动机浴轮㬌 机械密封浸渍石墨表面孔隙增大的原因是气相 $\mathrm{N}_{2} \mathrm{O}_{4}$ 在孔隙内部发生气蚀现象, 这种现象会使得 石墨变得松散。因此, 在冲击载荷、局部应力或局 部高压集中时静环石墨的表面在磨损时易于产生 大片的脱离。 


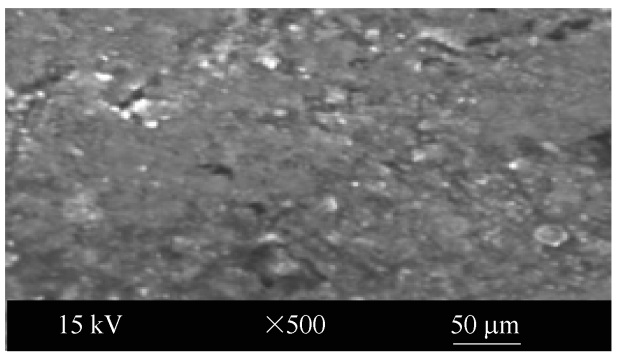

(a) 水介质磨损试验前

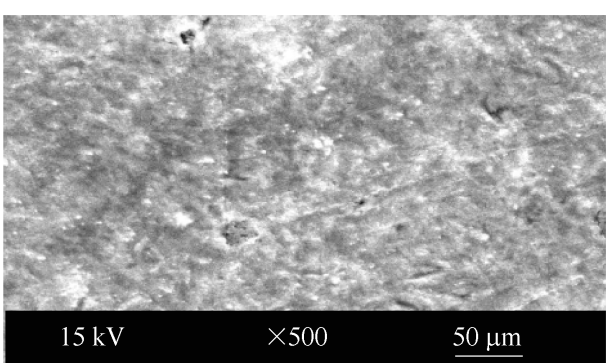

(b) 水介质磨损试验后

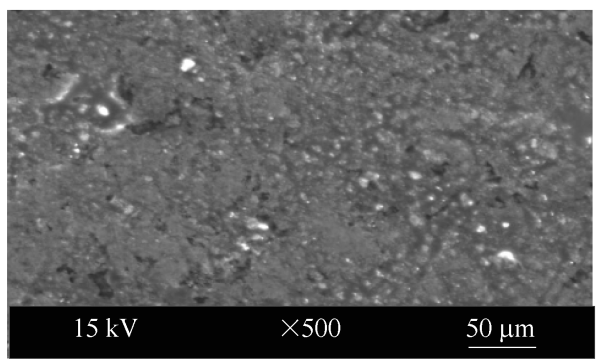

(c) $\mathrm{N}_{2} \mathrm{O}_{4}$ 介质磨损试验前

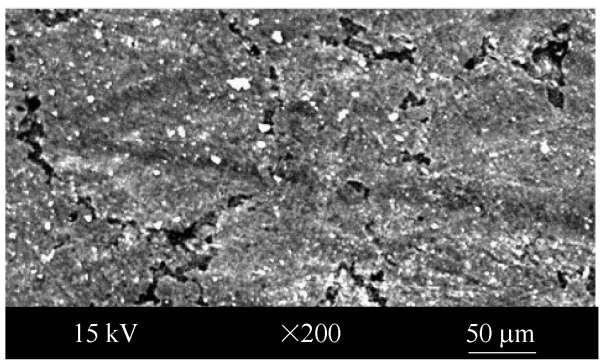

(d) $\mathrm{N}_{2} \mathrm{O}_{4}$ 介质磨损试验后

图 12 动态磨损试验电镜图

表 5 动态磨损石墨表面孔隙率

\begin{tabular}{ccc}
\hline 名称 & 表面孔隙率 $\xi(\%)$ & 气孔平均直径 $d / \mu \mathrm{m}$ \\
\hline 水介质磨损试验前 & 0.42 & 15 \\
水介质磨损试验后 & 0.37 & 16 \\
$\mathrm{~N}_{2} \mathrm{O}_{4}$ 介质磨损试验前 & 0.46 & 14 \\
$\mathrm{~N}_{2} \mathrm{O}_{4}$ 介质磨损试验后 & 5.60 & 33 \\
\hline
\end{tabular}

综合以上分析可以推断, 造成石墨表面孔隙增 多、孔径增大的原因是 $\mathrm{N}_{2} \mathrm{O}_{4}$ 气相对石墨孔隙的气 蚀, 即诱因 2 为导致石墨表面在 $\mathrm{N}_{2} \mathrm{O}$ 环境下磨损量 较大的主要原因。

\section{3 石墨制备工艺的改进措施}

从静态腐蚀以及动态磨损测试结果可知, 石墨 表面形貌的破坏不是来自 $\mathrm{N}_{2} \mathrm{O}_{4}$ 对树脂的腐蚀, 而是
由于气相 $\mathrm{N}_{2} \mathrm{O}_{4}$ 对石墨表面的气蚀。虽然, $\mathrm{N}_{2} \mathrm{O}_{4}$ 气 液两相共存的现象在浴轮葲工作时难以避免, 但可 以从石墨材料的性能改进入手，使得石墨表面能够 抵御气蚀的破坏, 这样石墨表面的严重磨损便不易 发生了。

在不改变其他条件的情况下，要想改善石墨表 面的气蚀问题，一种可行的做法是减小石墨材料的 表面孔隙率。图 13 为本文所采用的浸渍石墨制备 工艺流程图 ${ }^{[21]}$, 制备流程中影响石墨表面孔隙率的 环节有三个，分别是磨粉、成型和浸渍。

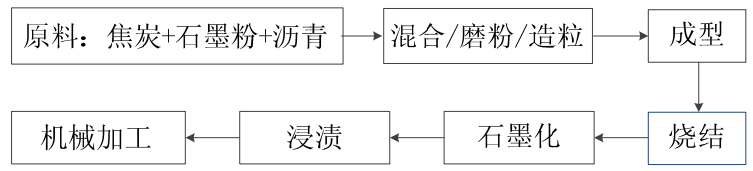

图 13 典型石墨制备工艺流程图

因此从改善石墨的材料本身性能入手，考虑到 石墨物理结构的特点，本文对石墨制备工艺提出以 下改进措施。(1) 从调整石墨原材料的颗粒度方面考 虑, 在对原材料进行磨粉加工时, 可减小其粉料的 颗粒度, 从目前的 200 目增加到 400 目, 粒径从原 来的 $75 \mu \mathrm{m}$ 减小到 $37 \mu \mathrm{m}$; (2) 在石墨毛坏压制环节, 适当提高压制压力; (3) 在酚醛树脂的浸渍环节, 适 当提高浸渍温度及浸渍压力, 并增长浸渍时间, 将 浸渍时间从目前的 $5 \mathrm{~h}$ 增加到 $10 \mathrm{~h}$ 以上。

\section{4 结论}

(1) 对机械密封石墨的磨损表面进行了扫描电 镜观测, 根据磨损的形貌对正常磨损区域与异常磨 损区域进行了区分，对导致异常磨损的诱因进行了 假设: 分别是树脂腐蚀和孔隙气蚀, 并建立了树脂 腐蚀模型与孔隙气蚀模型。

(2) 针对两个诱因的模型验证问题, 设计了静 态腐蚀与动态磨损的试验测试流程以验证树脂腐蚀 和孔隙气蚀的作用结果, 并分别在水及 $\mathrm{N}_{2} \mathrm{O}_{4}$ 环境下 进行了试验。

(3) 通过扫描电镜与红外光谱分析, 对静态腐 蚀及动态磨损的试验前后静环石墨表面的微观组织 特征结果进行了分析。结果表明 $\mathrm{N}_{2} \mathrm{O}_{4}$ 环境并不会造 成石墨浸渍物酚醛树脂的腐蚀, 造成石墨磨损量大 的诱因是气相 $\mathrm{N}_{2} \mathrm{O}_{4}$ 在石墨表面孔隙内部破裂导致 的气蚀, 这种现象会使得石墨变得松散。因此, 在 冲击载荷、局部应力或局部高压集中时静环石墨的 表面在磨损时易于产生大片的脱离。

（4）针对减少浸渍石墨在 $\mathrm{N}_{2} \mathrm{O}_{4}$ 环境下的气蚀 
问题, 提出了可减少表面孔隙的浸渍石墨制备工 艺的改进措施。本文的研究成果也可为其他高速旋 转机械的机械密封的石墨选材及制备工艺改进提供 参考。

\section{参 考 文 献}

[1] ZHANG Guoyuan, CHEN Guozhong, ZHAO Weigang. An experimental test on a cryogenic high-speed hydrodynamic non-contact mechanical seal[J]. Tribology Letters, 2017, 65 (3): 80-88.

[2] 张树强, 王良, 赵伟刚. 液体火箭发动机浴轮洜用机械 密封温度场及热载变形研究 [J]. 火箭推进, 2014,40(5): 92-98.

ZHANG Shuqiang, WANG Liang, ZHAO Weigang.

Research on temperature field and heat deformation ofmechanical seal in liquid rocket engine turbo pump[J]. Journal of Rocket Propulsion， 2014， 40(5): 92-98.

[3] 王建否. 含密封高速轴系的结构、力系和振动特性研究 [D]. 西安: 西安交通大学, 2014.

WANG Jianlei. Research on Structure, force system and vibration characteristice of high-speed shafting system with seal[D]. Xi'an: Xi'an Jiaotong University, 2014.

[4] 丁雪兴, 严如奇, 陈金林. 接触式机械密封摩擦界面温 度分布分形模型 [J]. 化工学报, 2014, 65(11): 4544-4550.

DING Xuexing, YAN Ruqi, CHEN Jinlin. Fractal model of temperature distribution of frictional interface in contacting mechanical seals[J]. CIESC Journal, 2014, 65(11): 4544-4550.

[5] 李克斯, 张尔卿, 傅攀. 不完备先验知识下的机械密封 端面磨损状态评估研究 [J]. 摩擦学学报, 2006, 36(6): 717-725.

LI Kesi, ZHANG Erqing, FU Pan. Condition assessment on mechanical seal face wear based on incomplete prior knowledge[J]. Tribology, 2006, 36(6): 717-725.

[6] 张晓东, 龙静, 郭志鸣. 基于 ANSYS 的机械密封环摩 擦磨损模拟[J]. 机械设计与制造，2008(2): 63-64.

ZHANG Xiaodong, LONG Jing, GUO Zhiming. Analysis on friction seal field of mechanical face seals using simulaition [J]. Machinery Design \& Manufacture, 2008(2): 63-64.

[7] 王悦昶, 刘芗, 黄伟峰. 过盈配合连接对核主泵机械密 封性能影响的 ANSYS 分析[J]. 机械工程学报, 2017, 53(5): 153-159.

WANG Yuechang, LIU Ying, HUANG Weifeng. ANSYS interference fit analysis of mechanical seals inreactor coolant pumps[J]. Journal of Mechanical Engineering, 2017, 53(5): 153-159.
[8] 赵伟刚, 张鹏鹏, 任姗姗. 液体火箭发动机涡轮洜机械 密封磨损机理研究 [J]. 火箭推进, 2017, 43(3): 10-16. ZHAO Weigang, ZHANG Pengpeng, REN Shanshan. Research on wear mechanism of mechanical seal for turbopump in liquid rocket engine[J]. Journal of Rocket Propulsion, 2017, 43(3): 10-16.

[9] 李双喜, 黄克松, 蔡纪宁, 等. 中压机械密封端面接触 状态和应力研究 [J]. 润滑与密封, 2016, 41(1)：10-14. LI Shuangxi, HUANG Kesong, CAI Jining, et al. Study on contact state and stress of medium pressure mechanical seal end face[J]. Lubrication \& Sealing, 2016, 41(1): $10-14$.

[10] WANG Jianlei, YAN Yudong, ZHANG Jian. Experimental study on friction and wear behaviour of amorphous carboncoatingsfor mechanical seals in cryogenic environment [J]. Applied Surface Science, 2012, 258: 9531-9535.

[11] JIA Qian, YUAN Xiaoyang, ZHANG Guoyuan. Dry friction and wear characteristics of impregnated graphite in a corrosive environment[J]. Chinese Journal of Mechanical Engineering, 2014, 27(5): 965-971.

[12] 李正大, 吴海英, 王少鹏. 影响石墨材料浸泡 $\mathrm{N}_{2} \mathrm{O}_{4}$ 后 密封性的研究分析[J]. 火箭推进, 2013, 39(3): 93-98. LI Zhengda, WU Haiying, WANG Shaopeng. Analysis of factors affecting sealing performance of graphite sealing material after soaking in $\mathrm{N}_{2} \mathrm{O}_{4}[\mathrm{~J}]$. Journal of Rocket Propulsion, 2013, 39(3): 93-98.

[13] 张远君, 王普光, 刘竹莹. 液体火箭发动机浴轮洜设计 [M]. 北京：北京航空航天大学出版社，1995.

ZHANG Yuanjun, WANG Puguang, LIU Zhuying . Design of turbopump for liquid rocket engine [D]. Beijing : Beijing University of Aeronautics and Astronautics Press, 1995.

[14] 王建否, 延育东, 张健. 高速机床主轴石墨轴承材料的 选型与性能评价[J]. 润滑与密封, 2017, 42(5): 86-91. WANG Jianlei, YAN Yudong, ZHANG Jian. Selection and performance evaluation of graphite materialsused for high-speed spindle bearings [J]. Lubrication Engineering, 2017, 42(5): 86-91.

[15] 贾谦. 巴氏合金与石墨轴承的制造工艺、质检与性能试 验研究[D]. 西安: 西安交通大学, 2017.

JIA Qian. Study on the manufacturing process, quality and performance tests of babbit and graphite bearing[D]. Xi'an: Xi'an Jiaotong University, 2017.

[16] 温诗铸, 黄平. 摩擦学原理 $[\mathrm{M}]$. 四版. 北京: 清华大学 出版社, 2012.

WEN Shizhu, HUANG Ping. Principles of Tribology [M]. Fourth Edition. Beijing: Tsinghua University Press, 2012. 
[17] 张帆, 李跃宗, 贾谦. 核主洜用水润滑石墨轴承制备 及性能检测的数据化研究 $[\mathrm{C}] / /$ 全国摩擦学大会, 中国, 兰州, 2013.

ZHANG Fan, LI Yaozong, JIA Qian. Study on the preparation and performance test of water lubricated graphite bearing for nuclear main pump[C]//National tribology Congress, China, Lanzhou, 2013.

[18] 张伟. 腐蚀条件下构件剩余寿命预测软件开发及材料 腐蚀数据获取[D]. 西安: 西安交通大学, 2009.

ZHANG Wei. The software development for forecast the residual life of components under the conditions of corrosion and corrosion data acquisition from experiments [D]. Xi'an: Xi'an Jiaotong University, 2009.

[19] 贾谦, 欧阳武, 张帆. 水润滑轴承磨损寿命预测校正试 验载荷的磁力模拟研究 [J]. 中国电机工程学报, 2014, 34(17): 2836-2842.

JIA Qian, OUYANG Wu, ZHANG Fan. Magnetic simulation research of prediction-correction test loading forwater-lubricated bearing wear life $[\mathrm{J}]$. Proceedings of the CSEE，2014，34(17): 2836-2842.

[20] 欧阳武, 张帆, 王建否. 低黏润滑轴承可靠性强化试验 中磁力加载研究 $[\mathrm{J}]$. 机械工程学报, 2015, 51(4): 199-205.

OUYANG Wu, ZHANG Fan, WANG Jianlei. Research on magnetic loading in reliability enhancement[J]. Journal of Mechanical Engineering, 2015, 51(4): 199-205.

[21] 孙玉霞, 李双喜, 李继和. 机械密封技术[M]. 北京: 化学工业出版社, 2014.

SUN Yuxia, LI Shuangxi, LI Jihe. Mechanical seal technology[M]. Beijing: Chemical Industry Press, 2014.

作者简介: 王建磊(通信作者), 男, 1978 年生, 博士, 讲师。主要研究 方向为密封-转子系统动力学、现代机械设计、摩擦学。

E-mail: jlwang@xaut.edu.cn

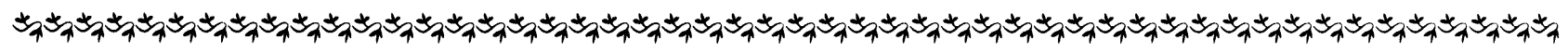

第 8 届上银优秀机械博士论文奖——佳作奖

\section{基于多视觉特征获取与融合的焊道轨迹自动识别}

作者: 曾锦乐

毕业学校: 清华大学

指导教师: 都东

在智能制造与精密焊接过程中，结合焊道区的多种视觉信息进行综合决策，充分利用不同视觉特征信息间的圥余性和互 补性准确识别焊道轨迹位置, 以实时补偿实际焊接轨迹与机器示教轨迹间的偏移, 对保证焊接产品质量具有重要意义。目前, 已有的焊道视觉检测技术大多仅针对焊道的单一视觉特征进行研究, 未能有效解决诸如航空航天等领域的强镜面反射表面工 件细隙焊道轨迹精密检测和能源等领域的中厚板多层多道焊接轨迹自动识别等问题。本文研究焊道多视觉特征同步获取与融 合方法, 旨在解决上述焊道轨迹检测难题。

本文研究了一种基于均匀光照图像和双线激光图像信息融合的焊道轨迹检测方法, 并将其应用于强镜面反射表面工件细 隙焊道轨迹识别场合。首先, 构建均匀光照条件克服镜面反射效应对成像质量的不利影响, 结合母材区和坡口区的反射特性 差异, 凸显图像中母材和焊道的灰度差异, 显著提高成像信噪比, 通过图像处理提取焊道轨迹的二维像素位置; 然后, 利用 双线激光光源投射工件表面，确定焊炬与工件表面的相对位姿关系；最后，采用 “双光源交替频闪-相机同步采集” 方式近同 步采集以上两种视觉信息, 并进行融合处理计算出细隙焊道的三维位姿信息, 实现了强镜面反射表面工件细隙焊道自动跟踪。

本文还研究了焊缝纹理特征、定向光影特征和结构光图像等多视觉信息同步获取与融合方法。首先, 研究了焊缝纹理特 征和定向光影特征的提取方法, 并将其初步应用于母材和焊缝边界识别场合; 然后, 结合以上成果, 采用 “多光源交替频闪 -相机同步采集” 方法近同步获取焊道表面的纹理特征、定向光影特征和结构光传感信息，分析不同视觉特征在焊道轨迹检测 场合的适用性, 最终提出一种基于多视觉特征时空域融合的焊道轨迹识别方法, 在焊接电弧干扰环境中实现了多层多道焊接 轨迹三维信息的准确识别。

本文研究成果已在航空航天制造、能源装备制造等领域开始得到应用, 显示出了良好前景, 为实现焊接过程智能控制提 供了基础。 